\title{
Glycaemic index and glycaemic load of selected popular foods consumed in Southeast Asia
}

\author{
Lijuan Sun, Davina Elizabeth Mei Lee, Wei Jie Kevin Tan, Dinesh Viren Ranawana, Yu Chin Rina Quek, \\ Hui Jen Goh and Christiani Jeyakumar Henry* $\dagger$ \\ Clinical Nutrition Research Centre, Singapore Institute for Clinical Sciences, A*STAR, Singapore
}

(Submitted 26 August 2014 - Final revision received 6 November 2014 - Accepted 9 December 2014 - First published online 26 February 2015)

\begin{abstract}
The objective of the present study was to determine the glycaemic index (GI) and glycaemic load (GL) values of standard portion sizes of Southeast Asian traditional foods. A total of fifteen popular Southeast Asian foods were evaluated. Of these foods, three were soft drinks, while the other twelve were solid foods commonly consumed in this region. In total, forty-seven healthy participants (eighteen males and twenty-nine females) volunteered to consume either glucose at least twice or one of the fifteen test foods after a $10-12 \mathrm{~h}$ overnight fast. Blood glucose concentrations were analysed before consumption of the test food, and 15, 30, 45, 60, 90 and 120 min after food consumption, using capillary blood samples. The GI value of each test food was calculated by expressing the incremental area under the blood glucose response curve (IAUC) value of the test food as a percentage of each participant's average IAUC value, with glucose as the reference food. Among the fifteen foods tested, six belonged to low-GI foods (Ice Green Tea, Beehoon, Pandan Waffle, Curry Puff, Youtiao and Kaya Butter Toast), three belonged to medium-GI foods (Barley Drink, Char Siew Pau and Nasi Lemak), and the other six belonged to high-GI foods (Ice Lemon Tea, Chinese Carrot Cake, Chinese Yam Cake, Chee Cheong Fun, Lo Mai Gai and Pink Rice Cake). The GI and GL values of these traditional foods provide valuable information to consumers, researchers and dietitians on the optimal food choice for glycaemic control. Moreover, our dataset provides GI values of fifteen foods that were not previously tested extensively, and it presents values of foods commonly consumed in Southeast Asia.
\end{abstract}

\section{Key words: Glycaemic index: Glycaemic load: Southeast Asia}

Currently, the Asian region has the largest rise in diabetes and associated chronic diseases. This is an outcome of a larger population size and changes in lifestyle and economic status. It has been estimated that diabetes and impaired glucose tolerance rates will increase by up to $60 \%$ by 2024 compared with the levels in $2007^{(1)}$. For example, the prevalence of type 2 diabetes in Singapore increased from 1.9\% in 1975 to $11.2 \%$ in 2010 , and is now one of the highest in the developed world ${ }^{(2)}$. Based on observational studies, a diet producing a low glycaemic response is associated with significantly less insulin resistance and a significantly lower prevalence of the metabolic syndrome ${ }^{(3)}$, the risk of type 2 diabetes $^{(4)}$ and the risk of coronary artery disease ${ }^{(5)}$ than a diet producing a high glycaemic response. Identifying lifestyle measures to improve insulin sensitivity and prevent diabetes in these Southeast Asian populations is particularly important. Although dietary interventions are the most effective and economical methods for the management and prevention of diabetes $^{(6)}$, there are currently limited data on the glycaemic responses of foods that are most commonly consumed in Southeast Asia.

Jenkins et al. ${ }^{(7)}$, more than two decades ago, introduced the glycaemic index (GI) as an alternative system for classifying carbohydrate-containing foods. The GI system ranks foods according to their effects on the postprandial glucose response. This system has been recommended to help guide food choice $\left(\mathrm{FAO}^{(8)}\right)$ because low-GI foods have been shown to improve blood glucose control in people with diabetes $^{(9)}$, leading to increased insulin sensitivity ${ }^{(10)}$ and $\beta$-cell function ${ }^{(11)}$. Low- and medium-GI foods have a lower impact on blood glucose levels, and are thus the preferred choice for glycaemic control. GI values represent the glycaemic response of isoglucidic foods, and therefore they are not always representative of the glycaemic effect of typical servings of those foods ${ }^{(12)}$. To quantify the overall glycaemic effect of a standard portion size of foods, the concept of

\footnotetext{
Abbreviations: GI, glycaemic index; GL, glycaemic load; IAUC, incremental area under the blood glucose response curve

*Corresponding author: Dr C. J. Henry, fax +656774 7134, email jeya_henry@sics.a-star.edu.sg

† Present address: Clinical Nutrition Research Centre, Singapore Institute for Clinical Sciences, A*STAR; Department of Biochemistry, Yong Loo Lin School of Medicine, National University of Singapore, 14 Medical Drive No. 07-02, MD 6 Building, Singapore 117599, Singapore.
} 
glycaemic load (GL) was introduced $^{(13)}$. It is often necessary to consider the GL along with GI values, especially when the carbohydrate content of the food is relatively small.

The GI value of a starchy food is determined by factors such as protein and fat content, degree of milling, degree of gelatinisation, fibre type and content, and processing and storage conditions $^{(14,15)}$. Consequently, there are often considerable variations in the GI value of the same food produced in different countries or by different manufacturers. Traditional Chinese or Southeast Asian foods are gaining in popularity, and are of increasing appeal worldwide. Publication of international GI values is needed for various ethnic food groups to ensure the availability of a comprehensive list for practical use. A recent review published up-to-date lists of GI values of more than 2000 different food items including a range of globally produced food groups and brands ${ }^{(16,17)}$. However, the vast majority of these published GI values are of Australian, British or Canadian origin, and some are of Danish, Indian and Japanese origin. Currently, only a few published GI and GL values are available for local Southeast Asian foods. Thus, it is necessary to determine the GI and GL values of Southeast Asian traditional foods in order to provide the local population with recommendations for an overall healthy diet.

\section{Methods}

\section{Subjects}

A total of forty-seven healthy participants (twenty-nine female and eighteen male) were recruited for the present study by means of advertisements, flyers and personal communication. Before inclusion into the study, potential participants were briefed on all aspects of the experiment and were given the opportunity to ask questions. Following the subjects' consent, a health assessment was performed, which included anthropometric measurements and a health questionnaire (giving details of food allergies/intolerance, metabolic diseases, special dietary needs, and smoking habits). Participants who met the following inclusion criteria were enrolled in the study: BMI $18.5-24.9 \mathrm{~kg} / \mathrm{m}^{2}$; systolic blood pressure $110-120 \mathrm{mmHg}$; diastolic blood pressure $75-85 \mathrm{mmHg}$; age $21-50$ years; fasting blood glucose $4-6 \mathrm{mmol} / \mathrm{l}$; not on prescription medication; non-smoker; no genetic or metabolic diseases. BMI and body fat were determined by the body composition analyser (BC-418; Tanita). Physical activity was quantified using the questionnaire of Baecke et al. ${ }^{(18)}$, and only those who did not participate in competitive sports and endurance events were included.

The present study was conducted according to the guidelines laid down in the Declaration of Helsinki, and all procedures involving human subjects were approved by the Domain Specific Review Board of National Healthcare Group. Written informed consent was obtained from all eligible subjects before participation

\section{Test foods}

A total of fifteen different foods were tested, including three soft drinks and twelve ready-to-eat prepared single foods or composite foods, namely (1) Ice Green Tea (Pokka Corporation (S) Private Limited); (2) Ice Lemon Tea (F\&N Foods Private Limited); (3) Barley Drink (F\&N Foods Private Limited); (4) Chinese Carrot Cake (Wei Cian Foods Private Limited); (5) Beehoon (NTUC FairPrice); (6) Chinese Yam Cake (Wei Cian Foods Private Limited); (7) Pandan Waffle (Dilys Creation); (8) Chee Cheong Fun (Hock Seng Food Private Limited); (9) Lo Mai Gai (Hock Seng Food Private Limited); (10) Pink Rice Cake (Dilys Creation); (11) Curry Puff (Old Chang Kee (S) Private Limited); (12) Char Siew Pau (Tanjong Rhu Pau \& Confectionery); (13) Youtiao (Koufu Private Limited); (14) Kaya Butter Toast (Just Acia Private Limited); (15) Nasi Lemak (Old Chang Kee (S) Private Limited). These foods represent a diverse range of commercial foods commonly consumed in Southeast Asia. A brief description of these foods is as follows: (1) Ice Green Tea: ready-to-drink brewed green tea; (2) Ice Lemon Tea: ready-to-drink black tea with lemon flavour; (3) Barley Drink: ready-to-drink packet drink made from pearl barley and dried winter melon; (4) Chinese Carrot Cake: turnip cake, daikon cake or radish cake, pan-fried shredded radish with plain rice flour (square-shaped slice); (5) Beehoon: rice vermicelli; (6) Chinese Yam Cake: taro cake or pan-fried shredded yam with plain rice flour (squareshaped slices); (7) Pandan Waffle: waffle batter prepared with pandan leaves, coconut milk, eggs and oil; (8) Chee Cheong Fun: rice noodle roll or steamed rice roll, plain served with sweet soya sauce; (9) Lo Mai Gai: steamed glutinous/sticky rice served with chicken meat; (10) Pink Rice Cake: teochew rice cake or Png Kueh, steamed rice pastry stuffed with pan-fried glutinous rice; (11) Curry Puff: deep-fried pastry dough stuffed with curry, chicken and potatoes; (12) Char Siew Pau: steamed barbecue pork bun; (13) Youtiao: deep-fried breadstick; (14) Kaya Butter Toast: toast with coconut jam and butter; (15) Nasi Lemak: steamed coconut milk rice with fried kuning fish, anchovies, peanuts, egg and spicy sambal sauce.

All the test foods and the reference food were given in portions containing $50 \mathrm{~g}$ of available carbohydrates. The glucose reference drink was made using $50 \mathrm{~g}$ of anhydrous glucose dissolved in $250 \mathrm{ml}$ of water at room temperature. Available carbohydrate values were provided by the manufacturer or tested in our laboratory (as described in the section 'Carbohydrate, protein and fat macronutrient analyses'). The nutrient composition of the three beverages was based on the manufacturer's data, while the remaining twelve test foods were analysed in our laboratory. The glucose and test foods were freshly prepared or bought from the local supermarket or food centre in the morning on test days, and served to the participants within $30 \mathrm{~min}$ of preparation. Beehoon was cooked in 1 litre of boiling water for $1.5 \mathrm{~min}$ and served. Beehoon was the only food that required cooking, and the rest of the test foods were ready-to-eat products. The nutrient compositions of the test foods are listed in Table 1.

\section{Study protocol}

The study was a randomised, controlled, cross-over, nonblinded design. The protocol used was adopted from that 
Table 1. Portion size and macronutrient composition of the test foods

\begin{tabular}{|c|c|c|c|c|c|}
\hline & Carbohydrate $(\mathrm{g} / 100 \mathrm{ml})$ & Experimental portion (ml) & Fat (g/portion) & Protein (g/portion) & Total energy (kJ/portion) \\
\hline \multicolumn{6}{|l|}{ Beverages } \\
\hline 1. Ice Green Tea & $6 \cdot 0$ & 833 & 0 & 0 & 837 \\
\hline 2. Ice Lemon Tea & $10 \cdot 0$ & 500 & 0 & 0 & 837 \\
\hline 3. Barley Drink & $7 \cdot 6$ & 658 & 0 & 2 & 854 \\
\hline Solid foods & Carbohydrate (g/100 g) & Experimental portion (g) & Fat (g/portion) & Protein (g/portion) & Total energy (kJ/portion) \\
\hline 4. Chinese Carrot Cake & 15.8 & 316.9 & 81.4 & 8.3 & 4042 \\
\hline 5. Beehoon & $80 \cdot 8$ & 61.9 & 1.0 & 3.5 & 933 \\
\hline 6. Chinese Yam Cake & $12 \cdot 8$ & 391.5 & $102 \cdot 6$ & $12 \cdot 6$ & 4908 \\
\hline 7. Pandan Waffle & $33 \cdot 7$ & $148 \cdot 3$ & $45 \cdot 5$ & $11 \cdot 0$ & 2736 \\
\hline 8. Chee Cheong Fun & $18 \cdot 0$ & $277 \cdot 4$ & $26 \cdot 9$ & $5 \cdot 2$ & 1937 \\
\hline 9. Lo Mai Gai & $28 \cdot 3$ & $176 \cdot 6$ & $19 \cdot 3$ & $9 \cdot 9$ & 1728 \\
\hline 10. Pink Rice Cake & $27 \cdot 1$ & $184 \cdot 8$ & $21 \cdot 2$ & $4 \cdot 3$ & 1707 \\
\hline 11. Curry Puff & $38 \cdot 6$ & $129 \cdot 6$ & $48 \cdot 9$ & $12 \cdot 7$ & 2887 \\
\hline 12. Char Siew Pau & $32 \cdot 3$ & $154 \cdot 7$ & $25 \cdot 7$ & $10 \cdot 8$ & 1987 \\
\hline 13. Youtiao & $45 \cdot 6$ & $109 \cdot 6$ & $35 \cdot 2$ & $7 \cdot 8$ & 2293 \\
\hline 14. Kaya Butter Toast & $46 \cdot 0$ & $108 \cdot 7$ & $17 \cdot 6$ & $7 \cdot 3$ & 1623 \\
\hline 15. Nasi Lemak & 27.9 & $179 \cdot 2$ & $25 \cdot 1$ & 14.4 & 2021 \\
\hline
\end{tabular}

described by Brouns et al. ${ }^{(19)}$, and is in line with procedures recommended by the $\mathrm{FAO} / \mathrm{WHO}^{(8)}$ for glycaemic response studies. According to the $\mathrm{FAO} / \mathrm{WHO}$, to determine the GI of a food, tests should be repeated in six or more subjects; moreover, it has been suggested that testing in ten subjects provides a degree of power and precision ${ }^{(19)}$. Thus, each test food was tested on at least ten subjects. At each session, subjects arrived at the Clinical Nutrition Research Centre laboratory at 08.30 hours, following an overnight fast of $10 \mathrm{~h}$. Subjects were advised to eat a meal of similar quantity and composition in the evening before the test session, and were asked to refrain from intensive physical activity. Upon arrival at the laboratory, subjects were first allowed to rest for $10 \mathrm{~min}$. Blood samples in the fasting state were then taken $5 \mathrm{~min}$ apart $(-5$ and 0$)$ for the analysis of blood glucose concentrations. Subsequently, subjects consumed the test/reference food along with $200 \mathrm{ml}$ of water at a comfortable pace, and finished it within $15 \mathrm{~min}$. Blood samples for glucose analysis were obtained at $15,30,45,60,90$ and 120 min intervals following the start of the meal. Participants were advised to keep physical activity to a minimum during testing, and remain seated. Computers, work tables, reading areas and a television were provided for their use. Upon completion of all the eight sessions, participants were debriefed and compensated accordingly for their time and travel expenses.

\section{Carbohydrate, protein and fat macronutrient analyses}

Total available carbohydrate content was analysed by an enzymatic method using a Megazyme assay kit (K-ACHDF; Megazyme International). Briefly, freeze-dried and defatted samples were incubated at $80^{\circ} \mathrm{C}$ with heat-stable $\alpha$-amylase to gelatinise, hydrolyse and depolymerise non-resistant starch, and then incubated at $60^{\circ} \mathrm{C}$ with protease (to solubilise and depolymerise proteins) and amyloglucosidase (to hydrolyse starch fragments to D-glucose). Then, the absorbance of the mixture was measured following enzymatic hydrolysis using a UV/visible spectrophotometer (UV-2600; Shimadzu) at a wavelength of $340 \mathrm{~nm}$ and $25^{\circ} \mathrm{C}$, for the determination of D-glucose and D-fructose. The percentage of total available carbohydrates (TAC) present in the food was derived from the following equation:

$$
\% \mathrm{TAC}=\% \text { D-glucose content }+\% \text { D-fructose content }
$$

Protein content was determined by the Kjeldahl method (Tecator $^{\mathrm{TM}}$ Digestor and Kjeltec ${ }^{\mathrm{TM}} 8200$ Auto Distillation Unit; FOSS), using a standard nitrogen-to-protein conversion factor of 6.25. Freeze-dried samples were used for all analyses. Fat content was determined gravimetrically by the solvent extraction method using the Soxtec System (Soxtec $^{\text {TM }} 2055$ Manual System; FOSS).

\section{Blood glucose analysis}

A qualified technician performed the blood glucose measurements. Blood was obtained by finger prick using the Accu-Chek, sterile, single-use, lancing device (Abbott). Recent reports have suggested that capillary rather than venous blood sampling is preferred for reliable GI testing ${ }^{(8,19,20)}$. Before a finger prick, subjects were encouraged to warm their hand to increase blood flow. To minimise plasma dilution, fingertips were not squeezed to extract blood, but were instead gently massaged, starting from the base of the hand and moving towards the tips. The first two drops of expressed blood were discarded, and the following drop was used for testing. Blood glucose concentration was measured using the HemoCue Glucose 201 Analyzer (HemoCue Glucose 201 RT). HemoCue is a reliable method for the analysis of blood glucose concentration ${ }^{(21)}$.

\section{Calculation of the glycaemic index and glycaemic load}

The total blood glucose response was expressed as the incremental area under the blood glucose response curve (IAUC), ignoring the area beneath the baseline, and was calculated geometrically using the trapezoidal rule ${ }^{(19,22)}$. The mean, standard deviation and CV of the IAUC of each subject's repeated 
reference food were calculated. The IAUC of each test food eaten by each subject was expressed as a percentage of the mean IAUC of the reference food eaten by the same subject:

$$
\mathrm{GI}=(\mathrm{IAUC} \text { test food } / \mathrm{IAUC} \text { reference food }) \times 100 \text {. }
$$

The GI value of each test food was taken as the mean for the whole group. The GL of a specific serving size of each food was calculated using the following equation:

$$
\begin{aligned}
\mathrm{GL}= & (\mathrm{GI} \text { of the test food } \\
& \times \text { available carbohydrate in a serving of the test food }(\mathrm{g})) / 100 .
\end{aligned}
$$

The serving size of each test food was taken from the information provided by the manufacturer.

\section{Data processing and statistical analysis}

Statistical analysis was performed using the Statistical Package for the Social Sciences (version 16.0; SPSS, Inc.), and data and figures were processed in a Microsoft Excel spreadsheet (2007). The GI values $>2$ sD above the mean were considered outliers and were excluded. Levels of inter- and intra-individual variations of the three standard (glucose) tests were assessed by determining the percentage of CV. Spearman's correlation coefficient $(\rho)$ was used to assess the relationship between the GI values and the nutrient content of each test food. Statistical significance was set at $P<0 \cdot 05$.

\section{Results}

The mean age of the participants was 23.3 (SEM 0.4) years (range 21-32 years). The mean BMI was $20 \cdot 8$ ( $\operatorname{sem~} 0 \cdot 2 \mathrm{~kg} / \mathrm{m}^{2}$ ) (range $18.5-24 \mathrm{~kg} / \mathrm{m}^{2}$ ). The mean body fat was 21.7 (SEM $\left.0 \cdot 2\right) \%$ (range 12.1-32\%). The average fasting blood glucose concentration was 4.5 (SEM 0.2$) \mathrm{mmol} / 1$ (range $4.1-5.0 \mathrm{mmol} / \mathrm{l}$ ).
The mean intra-individual CV of glycaemic responses to the three standard tests for the forty-seven participants was $16 \%$, which is consistent with recently reported data that low mean within-subject variation (reference $\mathrm{CV}<30 \%$ ) is required for accuracy ${ }^{(23)}$. The inter-individual variation in glycaemic responses to the standard test for all the forty-seven participants was $28.7 \%$.

The GI and GL values of all the tested foods are given in Table 2. The GI values are presented as means with their standard errors. For practical application, GI values are often grouped into categories of a low, medium or high glycaemic response. The cut-off values are as follows: low, $\leq 55$; medium 56-69 (inclusive); high, $\geq 70^{(24)}$. Of the test foods, six belonged to low-GI foods (Ice Green Tea, Beehoon, Pandan Waffle, Curry Puff, Youtiao and Kaya Butter Toast), three to medium-GI foods (Barley Drink, Char Siew Pau and Nasi Lemak), and the other six to high-GI foods (Ice Lemon Tea, Chinese Carrot Cake, Chinese Yam Cake, Chee Cheong Fun, Lo Mai Gai and Pink Rice Cake). From the following foods, one outlier was excluded: Barley Drink; Beehoon; Pink Rice Cake; Curry Puff; Youtiao; Kaya Butter Toast; Nasi Lemak.

There was no relationship between the GI value and the amount of protein per $50 \mathrm{~g}$ available carbohydrate portion (Spearman's $\rho=-0 \cdot 200$ ) and the amount of fat per $50 \mathrm{~g}$ available carbohydrate portion (Spearman's $\rho=-0.327$ ). This is in line with the previous findings of Henry et $a l^{(12,25)}$. Also, it was demonstrated that the amounts of protein and fat found in commonly consumed foods did not have a significant linear correlation with the glycaemic response.

\section{Discussion}

Most regions in Southeast Asia comprise three major ethnic groups, namely Chinese, Malay and Indian. There are many traditional and special foods, according to folk culture,

\begin{tabular}{|c|c|c|c|c|c|c|}
\hline & \multicolumn{2}{|c|}{ Gl } & \multirow{2}{*}{$\begin{array}{c}\text { Standard } \\
\text { serving size }(\mathrm{g})\end{array}$} & \multirow{2}{*}{$\begin{array}{l}\text { Carbohydrate } \\
\text { (g per serving) }\end{array}$} & \multirow[b]{2}{*}{ GL (per serving) } & \multirow{2}{*}{$\begin{array}{l}\text { Subjects } \\
(n)\end{array}$} \\
\hline & Mean & SEM & & & & \\
\hline \multicolumn{7}{|l|}{ Beverages } \\
\hline 1. Ice Green Tea & 50 & 5 & $250 \mathrm{ml}$ & $15 \cdot 0$ & 8 & 13 \\
\hline 2. Ice Lemon Tea & 74 & 7 & $250 \mathrm{ml}$ & $25 \cdot 0$ & 19 & 14 \\
\hline 3. Barley Drink & 62 & 6 & $250 \mathrm{ml}$ & $19 \cdot 0$ & 12 & 11 \\
\hline \multicolumn{7}{|l|}{ Solid foods } \\
\hline 4. Chinese Carrot Cake & 77 & 8 & 100 & $15 \cdot 8$ & 12 & 10 \\
\hline 5. Beehoon & 35 & 3 & 100 & $80 \cdot 8$ & 28 & 11 \\
\hline 6. Chinese Yam Cake & 86 & 11 & 120 & $15 \cdot 3$ & 13 & 10 \\
\hline 7. Pandan Waffle & 46 & 6 & 80 & $27 \cdot 0$ & 12 & 11 \\
\hline 8. Chee Cheong Fun & 81 & 7 & 200 & $36 \cdot 0$ & 29 & 10 \\
\hline 9. Lo Mai Gai & 106 & 12 & 160 & $45 \cdot 3$ & 48 & 12 \\
\hline 10. Pink Rice Cake & 97 & 12 & 130 & $35 \cdot 2$ & 34 & 11 \\
\hline 11. Curry Puff & 41 & 4 & 120 & $46 \cdot 3$ & 19 & 11 \\
\hline 12. Char Siew Pau & 66 & 7 & 130 & $42 \cdot 0$ & 28 & 10 \\
\hline 13. Youtiao & 55 & 4 & 100 & $45 \cdot 6$ & 25 & 11 \\
\hline 14. Kaya Butter Toast & 49 & 4 & 120 & $55 \cdot 2$ & 27 & 11 \\
\hline 15. Nasi Lemak & 66 & 5 & 280 & $78 \cdot 1$ & 52 & 12 \\
\hline
\end{tabular}

Table 2. Glycaemic index (Gl) and glycaemic load (GL) values of the test foods

(Mean values with their standard errors) 
religion and festival. Southeast Asian foods are quite different from Western foods; indeed, they are even different from the most authentic Chinese foods. There is little information about GI and GL values of Southeast Asian foods in the literature. Southeast Asian or Chinese cooking allows for a creative and stylistic touch, and it is one important reason why Southeast Asian or Chinese foods are always absent in the international GI and GL tables. In the present study, by using a recommended standard method, we determined the glycaemic values of three popular drinks and twelve foods commonly consumed in Southeast Asia. However, Nasi Lemak, being the only composite meal, was also tested using the same standard method as used for the other foods. This enabled consistency of methodology across all the test foods. Moreover, based on our previous study ${ }^{(26)}$, it has been shown that the calculated GI based on carbohydrate component produced vastly different GI values.

The GI values of the fifteen tested foods varied from 35 (Beehoon) to 109 (Lor Mai Gai). The GI of green tea was the lowest (50) among the three tested popular drinks. Based on the manufacturer's information, we have found that there are $250 \mathrm{mg}$ of tea polyphenols in green tea, which may have some influence on the glycaemic response. However, Josic et al. ${ }^{(27)}$ concluded that green tea showed no effect on the reduction in glucose and insulin levels when consumed with a breakfast meal consisting of white bread and turkey breast.

Rice noodle, which is made of rice (also known as Beehoon or rice vermicelli), has been tested in several studies. The GI value of dried rice noodles from Thailand was found to be 57 in Asian subjects ${ }^{(28)}$, Guilin rice noodles 37 and Jiangxi rice noodles 40 in UK subjects ${ }^{(29)}$, and Jianxi rice vermicelli 56 and Taiwan vermicelli 68 in subjects from Hong Kong ${ }^{(25)}$. The GI value of rice noodles tested in the present study was 35, which matched more closely with that of Guilin or Jiangxi rice noodles found in UK subjects. These variations might be due to differences in processing conditions and ingredients used in different countries. Therefore, this reconfirms the need to test food products in the country of consumption ${ }^{(17)}$.

A high GI value was obtained for Lo Mai Gai (109) and Pink Rice Cake (97), both made of glutinous rice that has been reported to have a consistently high GI value in previous studies $^{(17,30)}$. Chen et al. ${ }^{(30)}$ found that both fried bread stick and barbecue pork bun (in Hong Kong) had a higher GI value of 69 , compared with those found in the present study (55 and 64, respectively), although fat and protein levels were relatively similar.

The GI value of a single food is dependent on the composition of that food. Different elements can affect the GI of a food, such as the presence of $\mathrm{fat}^{(31)}$, $^{\left(10 t e i n^{(32)}\right.}$ and some anti-nutrients ${ }^{(14)}$. The presence of large amounts of protein or fat may significantly reduce the glycaemic response by increasing insulin secretion or slowing gastric emptying ${ }^{(33,34)}$. In our previous study, we found that either $30 \mathrm{~g}$ of groundnut oil or $15 \mathrm{~g}$ of chicken protein could lower the GI of white rice $^{(26)}$. The present study indicates that there was no significant correlation of protein or fat (macronutrients) with the GI. However, Jenkins et al. (7) reported that both fat and protein had a negative correlation with the GI, whereas Henry et al. ${ }^{(12)}$ found a negative correlation only between protein and the GI. A larger variety of test foods might be required for any correlation to be observed. Cooking methods such as steaming and stir-frying, which are more common in Asia than in Europe and America, may also influence the nutrient composition, and thus affect the glycaemic response.

The GL of a food allows a more representative comparison of glycaemic response after consumption of a portion of the food. As with the GI, a higher GL can indicate the likelihood of greater elevation in blood glucose concentration. However, the GL takes realistic portion sizes into account, which are to be consumed in normal circumstances ${ }^{(16)}$. Good examples would be the consumption of Chinese Carrot Cake and Chinese Yam Cake; although they have high GI values, their GL values are relatively lower in comparison with other lower-GI foods.

In summary, the results of the present study provide reliable values of the GI and GL for foods commonly consumed in Southeast Asia. The identification of Southeast Asian traditional foods with lower glycaemic responses may help lower the GI of the Southeast Asian diet, and potentially be beneficial for the Asian population in the management and prevention of chronic diseases such as obesity and diabetes.

\section{Acknowledgements}

The authors thank the volunteers who participated in the study.

The present study was supported by the Singapore Institute for Clinical Sciences.

The authors' responsibilities are as follows: L. S., D. E. M. L., W. J. K. T. and Y. C. R. Q. were responsible for the acquisition, analysis and interpretation of the data; D. V. R., Y. C. R. Q., H. J. G. and C. J. H. contributed to the conception and design of the study; L. S., D. E. M. L., W. J. K. T. and C. J. H. drafted the manuscript and critically revised the paper for important intellectual content. All authors read and approved the final manuscript.

The authors declare that there are no conflicts of interest.

\section{References}

1. Chan JC, Malik V, Jia W, et al. (2009) Diabetes in Asia: epidemiology, risk factors, and pathophysiology. JAMA $\mathbf{3 0 1}$ 2129-2140.

2. Soh SE, Tint MT, Gluckman PD, et al. (2013) Cohort profile: Growing Up in Singapore Towards healthy Outcomes (GUSTO) birth cohort study. Int J Epidemiol 43, 1401-1409.

3. McKeown NM, Meigs JB, Liu S, et al. (2004) Carbohydrate nutrition, insulin resistance, and the prevalence of the metabolic syndrome in the Framingham Offspring Cohort. Diabetes Care 27, 538-546.

4. Schulze MB, Liu S, Rimm EB, et al. (2004) Glycemic index, glycemic load, and dietary fiber intake and incidence of type 2 diabetes in younger and middle-aged women. $A m \mathrm{~J}$ Clin Nutr 80, 348-356.

5. Liu S, Willett WC, Stampfer MJ, et al. (2000) A prospective study of dietary glycemic load, carbohydrate intake, and 
risk of coronary heart disease in US women. Am J Clin Nutr 71, 1455-1461.

6. Kelley DE (2003) Sugars and starch in the nutritional management of diabetes mellitus. Am J Clin Nutr 78, 858S-864S.

7. Jenkins DJ, Wolever TM, Taylor RH, et al. (1981) Glycemic index of foods: a physiological basis for carbohydrate exchange. Am J Clin Nutr 34, 362-366.

8. FAO/WHO (1998) Carbohydrates in human nutrition. Report of a Joint FAO/WHO Expert Consultation. FAO Food Nutr Pap 66, 1-140.

9. Brand-Miller J, Hayne S, Petocz P, et al. (2003) Low-glycemic index diets in the management of diabetes: a meta-analysis of randomized controlled trials. Diabetes Care $\mathbf{2 6}$ 2261-2267.

10. Frost G, Leeds A, Trew G, et al. (1998) Insulin sensitivity in women at risk of coronary heart disease and the effect of a low glycemic diet. Metabolism 47, 1245-1251.

11. Wolever TM \& Mehling C (2002) High-carbohydrate-lowglycaemic index dietary advice improves glucose disposition index in subjects with impaired glucose tolerance. Br J Nutr 87, 477-487.

12. Henry CJ, Lightowler HJ, Strik CM, et al. (2005) Glycaemic index and glycaemic load values of commercially available products in the UK. Br J Nutr 94, 922-930.

13. Salmeron J, Ascherio A, Rimm EB, et al. (1997) Dietary fiber, glycemic load, and risk of NIDDM in men. Diabetes Care 20, $545-550$

14. Thorne MJ, Thompson LU \& Jenkins DJ (1983) Factors affecting starch digestibility and the glycemic response with special reference to legumes. Am J Clin Nutr 38, 481-488.

15. Bjorck I, Granfeldt Y, Liljeberg H, et al. (1994) Food properties affecting the digestion and absorption of carbohydrates. Am J Clin Nutr 59, 699S-705S.

16. Foster-Powell K, Holt SH \& Brand-Miller JC (2002) International table of glycemic index and glycemic load values: 2002. Am J Clin Nutr 76, 5-56.

17. Atkinson FS, Foster-Powell K \& Brand-Miller JC (2008) International tables of glycemic index and glycemic load values: 2008. Diabetes Care 31, 2281-2283.

18. Baecke JA, Burema J \& Frijters JE (1982) A short questionnaire for the measurement of habitual physical activity in epidemiological studies. Am J Clin Nutr 36, 936-942.

19. Brouns F, Bjorck I, Frayn KN, et al. (2005) Glycaemic index methodology. Nutr Res Rev 18, 145-171.

20. Wolever TM, Vorster HH, Bjorck I, et al. (2003) Determination of the glycaemic index of foods: interlaboratory study. Eur J Clin Nutr 57, 475-482.

21. Stork AD, Kemperman H, Erkelens DW, et al. (2005) Comparison of the accuracy of the HemoCue glucose analyzer with the Yellow Springs Instrument glucose oxidase analyzer, particularly in hypoglycemia. Eur $J$ Endocrinol 153 , $275-281$.

22. Allison DB, Paultre F, Maggio C, et al. (1995) The use of areas under curves in diabetes research. Diabetes Care 18 245-250.

23. Wolever TM, Brand-Miller JC, Abernethy J, et al. (2008) Measuring the glycemic index of foods: interlaboratory study. Am J Clin Nutr 87, 247S-257S.

24. Brand-Miller J, Wolover TMS, Foster-Powell K, et al. (2003) The New Glucose Revolution: The Authoritative Guide to the Glycemic Index. New York: Marlowe \& Company.

25. Lok KY, Chan R, Chan D, et al. (2010) Glycaemic index and glycaemic load values of a selection of popular foods consumed in Hong Kong. Br J Nutr 103, 556-560.

26. Sun L, Ranawana DV, Leow MK, et al. (2014) Effect of chicken, fat and vegetable on glycaemia and insulinaemia to a white rice-based meal in healthy adults. Eur J Nutr $\mathbf{5 3}$, $1719-1726$.

27. Josic J, Olsson A, Wickeberg J, et al. (2010) Does green tea affect postprandial glucose, insulin and satiety in healthy subjects: a randomized controlled trial. Nutr J 9, 63.

28. Chan HM, Brand-Miller JC, Holt SH, et al. (2001) The glycaemic index values of Vietnamese foods. Eur J Clin Nutr $\mathbf{5 5}$, 1076-1083.

29. Ranawana DV, Henry CJ, Lightowler HJ, et al. (2009) Glycaemic index of some commercially available rice and rice products in Great Britain. Int J Food Sci Nutr 60, Suppl. 4, 99-110.

30. Chen YJ, Sun FH, Wong SH, et al. (2010) Glycemic index and glycemic load of selected Chinese traditional foods. World J Gastroenterol 16, 1512-1517.

31. Collier G \& O'Dea K (1983) The effect of coingestion of fat on the glucose, insulin, and gastric inhibitory polypeptide responses to carbohydrate and protein. Am J Clin Nutr 37, 941-944.

32. Granfeldt Y, Bjorck I \& Hagander B (1991) On the importance of processing conditions, product thickness and egg addition for the glycaemic and hormonal responses to pasta: a comparison with bread made from 'pasta ingredients'. Eur J Clin Nutr 45, 489-499.

33. Collier G, McLean A \& O'Dea K (1984) Effect of co-ingestion of fat on the metabolic responses to slowly and rapidly absorbed carbohydrates. Diabetologia 26, 50-54.

34. Nuttall FQ, Mooradian AD, Gannon MC, et al. (1984) Effect of protein ingestion on the glucose and insulin response to a standardized oral glucose load. Diabetes Care 7, 465-470. 